\title{
Trypanosoma rangeli: un protozoo infectivo y no patógeno para el humano que contribuye al entendimiento de la transmisión vectorial y la infección por Trypanosoma cruzi, agente causal de la enfermedad de $C$ hagas
}

\author{
G ustavo A dolfo Vallejo*,Yazmín Suárez, J enny Lorena Olaya, Sneider A lexander G utiérrez, J ulio César C arranza
}

Laboratorio de Investigaciones en Parasitología Tropical (LIPT), Facultad de Ciencias, Universidad del Tolima, Ibagué, Colombia

\begin{abstract}
Resumen
A diferencia de Trypanosoma cruzi, agente causal de la enfermedad de Chagas, Trypanosoma rangeli es un parásito infectivo y no patógeno para el humano, pero sí para los vectores del género Rhodnius. Con base en polimorfismos del ADN del cinetoplasto (kADN), del gen miniexón o líder de empalme (Spliced Leader), del espaciador interno transcrito (ITS) y de la subunidad pequeña del ribosoma (SSU rARN), se han descrito varios genotipos de T. rangeli (KP1+/A, B, KP1-/C, KP1-/D y E), los cuales son transmitidos selectivamente por dos líneas filogenéticas de Rhodnius, lo que indica la ocurrencia de procesos coevolutivos entre el parásito y los vectores. La línea Robustus (Rhodnius prolixus, Rhodnius robustus y Rhodnius neglectus) transmite exclusivamente el genotipo KP1+/A y la línea Pallescens (Rhodnius pallescens, Rhodnius colombiensis y Rhodnius ecuadoriensis) transmite el genotipo KP1-/C. Aunque el conocimiento de las bases moleculares de la interacción parásito-vector es todavía escaso, este trabajo presenta observaciones inéditas sobre la capacidad de los genotipos KP1+/A y KP1-/C para completar el ciclo de vida en algunas de las 19 especies de Rhodnius y la detección de factores tripanolíticos en $R$. prolixus, $R$. robustus y $R$. neglectus contra el genotipo KP1-/C y varios genotipos de T. cruzi. Los avances recientes en los estudios de transcripción genómica de $T$. rangeli, y su comparación con $T$. cruzi constituyen el punto de partida para entender cabalmente la transmisión vectorial selectiva de T. rangeli y T. cruzi, así como de la patogenia de T. rangeli para el vector y de la incapacidad de $T$. rangeli y la capacidad de $T$. cruzi para invadir células del mamífero.
\end{abstract}

Palabras clave: Trypanosoma rangeli KP1(+), Trypanosoma rangeli KP1(-), epidemiología molecular, genoma de T. rangeli, Rhodnius spp.

Trypanosoma rangeli: an infective but non-pathogenic protozoon for humans which contributes to the understanding of the vector-borne transmission and the pathogenesis of Trypanosoma cruzi, causative agent of Chagas' disease

\begin{abstract}
Unlike Trypanosoma cruzi, the causative agent of Chagas' disease, T. rangeli is an infective, non-pathogenic parasite for humans, but pathogenic for vectors from the Rhodnius genus. Several T. rangeli genotypes (KP1+/A, B, KP1-/C, KP1-/D and E) have been described based on kinetoplast DNA (kDNA) polymorphisms, the spliced leader or miniexon, the intergenic transcribed spacer (ITS) and the small ribosomal subunit (SSUrRNA). These are selectively transmitted by two Rhodnius phylogenetic lines, thereby indicating co-evolutionary processes between parasite and vector genotypes. The Robustus line (Rhodnius prolixus, $R$. robustus and $R$. neglectus) exclusively transmits the KP1+/A genotype and the Pallescens line ( $R$. pallescens, $R$. colombiensis and $R$. ecuadoriensis) only transmits the KP1-/C genotype. Even though little knowledge is available regarding the molecular basis of parasitevector interaction, the present work presents unpublished observations about KP1+/A and KP1-/C genotype ability to complete the life-cycle of some of the 19 Rhodnius species and the detection of trypanolytic factors in R. prolixus, $R$. robustus and $R$. neglectus against the KP1-/C genotype and some T. cruzi genotypes. Several advances regarding molecular, transcriptome and genomic studies dealing with $T$. rangeli are presented and compared to T. cruzi; these are the starting point for understanding the selective vectorial transmission of $T$. rangeli and $T$. cruzi, T. rangeli pathogenicity for the vector, as well as T. rangeli inability and T. cruzi ability to invade mammalian cells.
\end{abstract}

Key words: Trypanosoma rangeli KP1(+), Trypanosoma rangeli KP1(-), molecular epidemiology, T. rangeli genome, Rhodnius spp. 


\section{Introducción}

Trypanosoma rangeli fue reportado por primera vez en Colombia en Rhodnius prolixus del municipio de Prado, Tolima (Uribe, 1929). Los estudios sobre el ciclo del parásito en el vector, su persistencia e inocuidad en los humanos se deben a Groot, et al. $(1950,1951)$ y a Groot (1952, 1954). Algunos de los estudios posteriores sobre la biología y la epidemiología de $T$. rangeli fueron llevados a cabo por M arinkelle (1968a, 1968b); D 'Alessandro, 1976; D’A lesandro \& G ore-Saravia, 1999; G uhl \& M arinkelle, 1982, y G uhl, et al., 1985. T. rangeli y Trypanosoma cruzi son las dos únicas especies de tripanosomas que infectan al hombre en los países de América Latina. T. cruzi es el agente causal de la enfermedad de Chagas, la cual afecta a entre 7 y 8 millones de personas en las zonas endémicas, en tanto que alrededor de 60 millones se encuentran en riesgo de adquirir la infección desde el sur de los Estados Unidos hasta el sur de Argentina y Chile (Organización Panamericana de la Salud, 2007; Organización M undial de la Salud, 2014). Aunque las infecciones humanas por T. rangeli son frecuentes, se considera que no es patógeno para el vertebrado, pero sí para las especies del género Rhodnius, las cuales son los vectores comprobados de este parásito (G uhl $\boldsymbol{\&}$ Vallejo, 2003). En algunos países de América Latina las dos especies de tripanosomas se encuentran infectando a los mismos vertebrados y vectores, lo cual dificulta el acertado diagnóstico e identificación del agente causal de la enfermedad de Chagas (Vallejo, et al., 1988). Es por esto que los estudios de T. rangeli se enmarcan dentro de la epidemiología de la enfermedad de Chagas; en los últimos años, $T$. rangeli se ha considerado como un modelo para los estudios biológicos y moleculares relacionados con las interacciones parásito-hospedero-vector, pues su conocimiento ayudará a entender la dinámica de la transmisión vectorial de $T$. cruzi y de $T$. rangeli, las bases químicas y moleculares de la patogénesis de $T$. cruzi y la ausencia de patogenia de $T$. rangeli para el vertebrado, lo cual podría conducir a la identificación de blancos terapéuticos y al desarrollo de drogas o de vacunas para el tratamiento de la enfermedad de Chagas.

\section{Interacción parásito-vector en T. rangeli}

Se han reconocido cuatro componentes en la interacción parásito-vector que son determinantes en la transmisión de los tripanosomas. Estos componentes son: i) la especie, la cepa y el genotipo del tripanosoma; ii) la especie de triatomino; iii) la respuesta inmune celular y humoral del vector, y iv) la microbiota intestinal del insecto (Schaub, 2009; G arcía, et al., 2010; G arcía, et al., 2012).

\footnotetext{
* Correspondencia:

Gustavo Adolfo Vallejo; gvallejo@ut.edu.co

Recibido: 29 de octubre de 2014

Aceptado: 9 de febrero de 2015
}

Los vectores de $T$. cruzi y $T$. rangeli se infectan cuando se alimentan de sangre de mamíferos que están parasitados. A diferencia de $T$. cruzi, que es transmitido a través de las heces del vector, T. rangeli pasa del intestino a la hemolinfa, invade las glándulas salivares y se transmite a través de la picadura del insecto (G root, 1954; D'A lessandro, 1976). Aunque T. rangeli puede infectar el intestino de cualquier especie de triatomino, los únicos vectores en los cuales hay invasión de las glándulas salivares y producción de tripomastigotes metacíclicos infectivos para el mamífero son las 19 especies hasta ahora reconocidas del género Rhodnius (A bad-Franch, et al., 2013; da Rosa, et al., 2014). En 13 de estas 19 especies se ha verificado la infección natural o experimental por $T$. rangeli (Tabla 1 ).

Cuando T. rangeli ingresa al intestino del vector, se enfrenta a simbiontes y bacterias comensales, factores hemolíticos, factores tripanolíticos y lectinas; luego, el parásito se multiplica en el intestino produciendo formas similares a los amastigotes, los epimastigotes cortos, los epimastigotes largos, los esferomastigotes y los tripomastigotes (Vallejo, et al., 1988).

Después, los epimastigotes cortos atraviesan la pared intestinal y se enfrentan en la hemolinfa del insecto a los componentes del sistema inmune como las lisozimas, el sistema de la profenoloxidasa (proFO), los factores tripanolíticos, la producción de superóxido y óxido nítrico, la fagocitosis mediada por hemocitos, entre otros (G arcía, et al., 2009). Estos epimastigotes cortos invaden los hemocitos y se multiplican dentro de ellos, y luego son reemplazados por epimastigotes largos, los cuales se adhieren a las glándulas salivares y penetran en ellas para finalmente producir los tripomastigotes metacíclicos, que son las formas infectivas para el mamífero (Figura 1).

Varios trabajos resumidos por Azambuja \& G arcía, 2005; Azambuja, et al., 2005; G arcía, et al., 2009, y G arcía, et al., 2012, han demostrado que el éxito de la infección de $T$. rangeli se debe a que una vez el parásito ha alcanzado el hemocele del insecto, produce una inhibición de la respuesta inmune celular y humoral, lo que permite la multiplicación del parásito en la hemolinfa, la invasión de hemocitos, la invasión de las glándulas salivares y la producción de tripomastigotes metacíclicos o formas infectivas.

\section{G enotipos de T. rangeli y su asociación con vectores del género Rhodnius}

Existen cepas de $T$. rangeli que no logran sobrevivir a la respuesta inmune del vector o no consiguen producir formas infectivas en las glándulas salivares. También se reconoce que las especies de Rhodnius son susceptibles a la infección de cepas de $T$. rangeli de la misma región geográfica y no de otras regiones. En este sentido, se ha reportado que las cepas de $T$. rangeli de aislamientos procedentes de Colombia y Costa Rica no generaron tripomastigotes metacíclicos en $R$. pallescens de Panamá o R. ecuadoriensis de Ecuador o de 
Tabla 1. Genotipos de T. rangeli aislados de glándulas salivares en infecciones naturales o experimentales en las especies de Rhodnius

\begin{tabular}{|c|c|c|c|c|}
\hline Rhodnius spp & $\begin{array}{l}\text { Infección natural } \\
\text { o experimental }\end{array}$ & kADN & $\begin{array}{l}\text { SSU } \\
\text { rARN }\end{array}$ & R eferencia \\
\hline R. amazonicus & ND & ND & ND & - \\
\hline R. barretti & ND & ND & ND & - \\
\hline R. brethesi & $\mathrm{N}$ & ND & $\mathrm{B}$ & M aia da Silva, et al., 2007 \\
\hline R. colombiensis & $\mathrm{N}$ & KP1(-) & $\mathrm{C}$ & Vallejo, et al., 2002, 2009a \\
\hline R. dalessandroi & $\mathrm{N}$ & ND & ND & D 'Alessandro \& Saravia, 1999 \\
\hline$R$. domesticus & $\mathrm{E}$ & $\mathrm{KP} 1(+)$ & A & M achado, et al., 2001 \\
\hline R. ecuadoriensis & $\mathrm{N}$ & KP1(-) & $\mathrm{C}$ & Vallejo, et al., 2003, 2009a \\
\hline R. milesi & ND & ND & ND & - \\
\hline R. montenegrensis & $\mathrm{N}$ & ND & ND & Meneguetti, et al. , 2014 \\
\hline R. nasutus & $\mathrm{E}$ & ND & ND & M achado, et al., 2001 \\
\hline R. neglectus & $\mathrm{N}$ & $\mathrm{KP} 1(+)$ & A & M achado, et al., 2001 \\
\hline R. neivai & $\mathrm{N}$ & ND & ND & D ‘Alessandro \& H incapié, 1986 \\
\hline R. pallescens & $\mathrm{N}$ & KP1(-) & $\mathrm{C}$ & Vallejo, et al., 2003, 2009a; Urrea, et al., 2011 \\
\hline R. paraensis & ND & ND & ND & - \\
\hline R. pictipes & $\mathrm{N}$ & ND & ND & D ‘Alessandro \& Saravia, 1999 \\
\hline R. prolixus & $\mathrm{N}$ & $\mathrm{KP} 1(+)$ & A & $\begin{array}{l}\text { Vallejo, et al., 2002, 2009a; Maia da Silva, } \\
\text { et al., 2007; Urrea, et al., } 2011\end{array}$ \\
\hline R. robustus & $\mathrm{N}$ & $\mathrm{KP} 1(+)$ & A & $\begin{array}{l}\text { M aia da Silva, et al., 2007; Vallejo, et al., } \\
\text { 2009a; Urrea, et al., } 2011\end{array}$ \\
\hline R. stali & ND & ND & ND & - \\
\hline R. zeledoni & ND & ND & ND & - \\
\hline
\end{tabular}

$\mathrm{N}$ = infección natural; E= infección experimental; ND = no determinada

Perú (D 'Alessandro, 1976). Asimismo, las cepas aisladas de $R$. colombiensis no infectaron a $R$. prolixus de Colombia (Vallejo, et al., 2002).

El estudio de esta restricción biológica existente entre $T$. rangeli y algunas especies de Rhodnius comenzó con el análisis de las cepas de $T$. rangeli aisladas de dos especies simpátricas, $R$. colombiensis y $R$. prolixus, recolectadas en el municipio de Coyaima, Tolima, en la región central de Colombia. $R$. prolixus es una especie domiciliada y $R$. colombiensis es un vector silvestre que se ha encontrado en esta región visitando las viviendas humanas sin dar lugar a colonias permanentes del insecto. Mediante la caracterización molecular del ADN del cinetoplasto se logró identificar dos genotipos de $T$. rangeli denominados $T$. rangeli $\mathrm{KP} 1(+) \mathrm{y}$ T. rangeli KP1(-) (Figura 2). Todas las cepas de T. rangeli aisladas de las glándulas salivares de $R$. colombiensis presentaron el perfil KP1(-) y las aisladas de $R$. prolixus fueron caracterizadas como KP1(+) (Vallejo, et al., 2002). Además, se hizo la caracterización molecular de $T$. rangeli en 815 ejemplares de $R$. prolixus de diferentes regiones geográficas de Colombia y, mediante PCR, se detectaron en el intestino 24 cepas KP1(-) y $27 \mathrm{KP} 1(+)$, en tanto que las 13 aisladas de las glándulas salivares mostraron el genotipo KP1(+). Por otro lado, la caracterización molecular de $T$. rangeli en 413 ejemplares de $R$. colombiensis permitió detectar por PCR en el intestino $104 \mathrm{KP} 1(-)$ y 6 KP1(+), en tanto que las 22 cepas aisladas de las glándulas salivares presentaron el genotipo KP1(-) (datos no publicados). Estos resultados sugieren que ambas especies de Rhodnius se pueden infectar en el intestino con los genotipos KP1(+) y KP1(-), pero que $R$. prolixus solo transmite KP1(+) y $R$. colombiensis transmite KP1(-) a través de las glándulas salivares.

En nuevos aislamientos de $T$. rangeli obtenidos a partir de $R$. pallescens, $R$. colombiensis, $R$. ecuadoriensis, $R$. prolixus y $R$. neglectus, se encontró que las especies de la cordillera de los Andes (R. pallescens, $R$. colombiensis, $R$. ecuadoriensis) transmiten el genotipo KP1(-), mientras que $R$. prolixus y $R$. neglectus, ubicadas principalmente al oriente de la cordillera de los Andes, transmiten el genotipo KP1(+) (Urrea, et al., 2005). 
Infección productiva de T. rangeli en Rhodnius spp
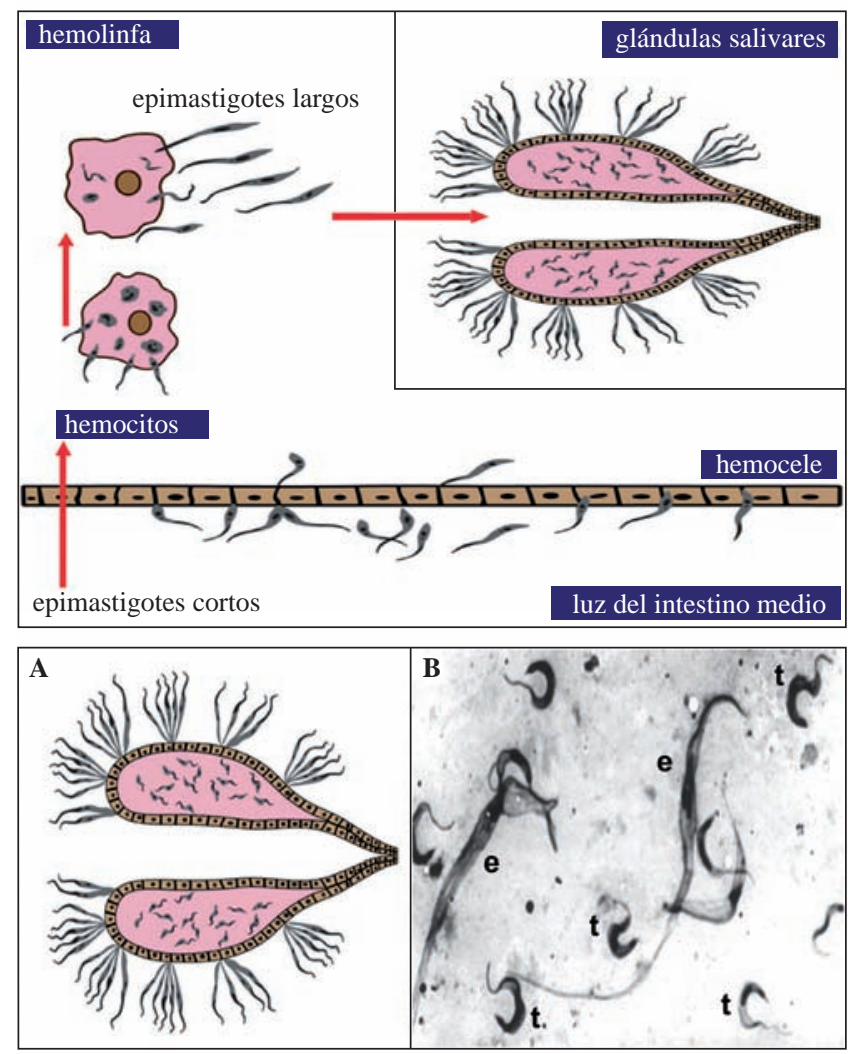

Figura 1. A. Diagrama esquemático que muestra los epimastigotes largos (e) de T. rangeli adheridos a la superficie de las glándulas salivares y los tripomastigotes metacíclicos (t) en la luz de las glándulas salivares. B. Epimastigotes largos (e) y tripanomastigotes metacíclios (t) coloreados con Giemsa (x1500) (Vallejo, 1984)

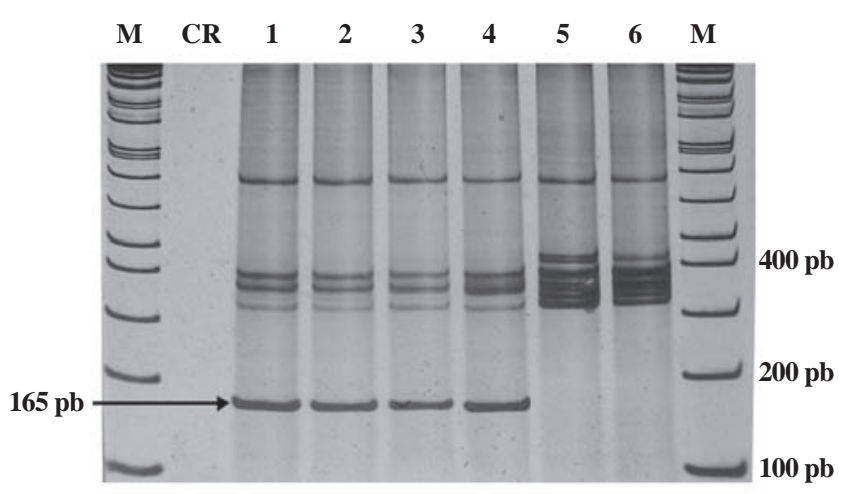

Figura 2. Genotipos de T. rangeli obtenidos mediante amplificación del kADN utilizando PCR dúplex (Vallejo, et al., 2002). Canaletas 1-4: cepas de T. rangeli KP1(+) aisladas de glándulas salivares de $R$. prolixus. Canaletas 5-6: T. rangeli KP1(-) aisladas de glándulas salivares de $R$. colombiensis. Nótese que el genotipo KP1(-) carece de la banda inferior de 165 pb derivada del minicírculo KP1, banda que solo está presente en el genotipo KP1(+). CR: control de reacción sin ADN. M: marcador de tamaño molecular, 1 Kb Plus DNA Ladder.
En los años siguientes, el estudio de la variabilidad de diferentes marcadores moleculares generó un aumento en la nomenclatura de T. rangeli. Así, la variabilidad del miniexón o líder de empalme (Spliced Leader) permitió describir nuevos genotipos denominados A, B, C, D y E (Maia da Silva, et al., 2004a, 2004b, 2007, 2009). El análisis de 101 cepas de $T$. rangeli correspondientes a diferentes genotipos, y su asociación con especies de Rhodnius, confirmó que el genotipo C corresponde a KP1(-), habiéndose aislado de $R$. pallescens, $R$. colombiensis y $R$. ecuadoriensis. El genotipo B fue aislado de $R$. brethesi, pero no se ha verificado si es $\mathrm{KP} 1(+)$ o KP1(-). El genotipo A corresponde a KP1(+) y fue aislado de $R$. prolixus, $R$. robustus y $R$. neglectus (Vallejo, et al., 2002, 2003; Vallej 0, et al., 2009a; Vallej 0, et al., 2009b; Urrea, et al., 2005, 2011) (Figura 3). En Centroamérica las cepas de $T$. rangeli $\mathrm{KP} 1(+)$ se aislaron en áreas de distribución de $R$. prolixus y las cepas de T. rangeli KP1(-), en áreas de distribución de $R$. pallescens (Salazar-Antón, et al., 2009).

De acuerdo con Schofield \& Dujardin (1999) y A badF ranch, et al., (2009), se han reconocido dos líneas filogenéticas principales en las especies de Rhodnius, denominadas la línea Pallescens y la línea Robustus. El genotipo KP1(-) se encuentra asociado a $R$. pallescens, $R$. colombiensis $y$ $R$. ecuadoriensis (línea Pallescens) y el genotipo KP1(+), a $R$. prolixus, $R$. robustus, $R$. neglectus (línea Robustus), lo que indica la ocurrencia de procesos coevolutivos entre los genotipos de los parásitos y los vectores (Urrea, et al., 2005, 2011). Se espera que T. rangeli KP1(+) se detecte en infecciones naturales en $R$. domesticus y en $R$. nasutus, especies pertenecientes a la línea Robustus. Aún deben identificarse los genotipos de T. rangeli transmitidos naturalmente por 11 de las 19 especies de Rhodnius, lo cual contribuiría a confirmar la presencia de procesos coevolutivos en estas especies de vectores. La tabla 1 muestra la caracterización molecular de las cepas de $T$. rangeli aisladas de glándulas salivares en infecciones naturales o experimentales de Rhodnius spp.

\section{Actividad tripanolítica de la hemolinfa de $R$. prolixus y $R$. robustus contra genotipos de $T$. rangeli}

Después de analizar más de 100 cepas de T. rangeli aisladas de glándulas salivares de Rhodnius spp, nunca se encontró a $R$. prolixus con $T$. rangeli $\mathrm{KP} 1(-)$ ni a $R$. pallescens, $R$. colombiensis o $R$. ecuadoriensis con KP1(+). Esto llevó a sospechar la existencia de factores inhibitorios contra genotipos de $T$. rangeli presentes en la hemolinfa o en las glándulas salivares de los vectores.

La hemolinfa de $R$. prolixus y $R$. robustus, libre de hemocitos, se ha incubado con los genotipos de T. rangeli, encontrándose actividad tripanolítica contra epimastigotes de KP1(-) aislados de $R$. colombiensis, $R$. pallescens y $R$. ecuadoriensis, pero no contra epimastigotes KP1(+) (Pulido, et al., 2008). Recientemente hemos encontrado en 


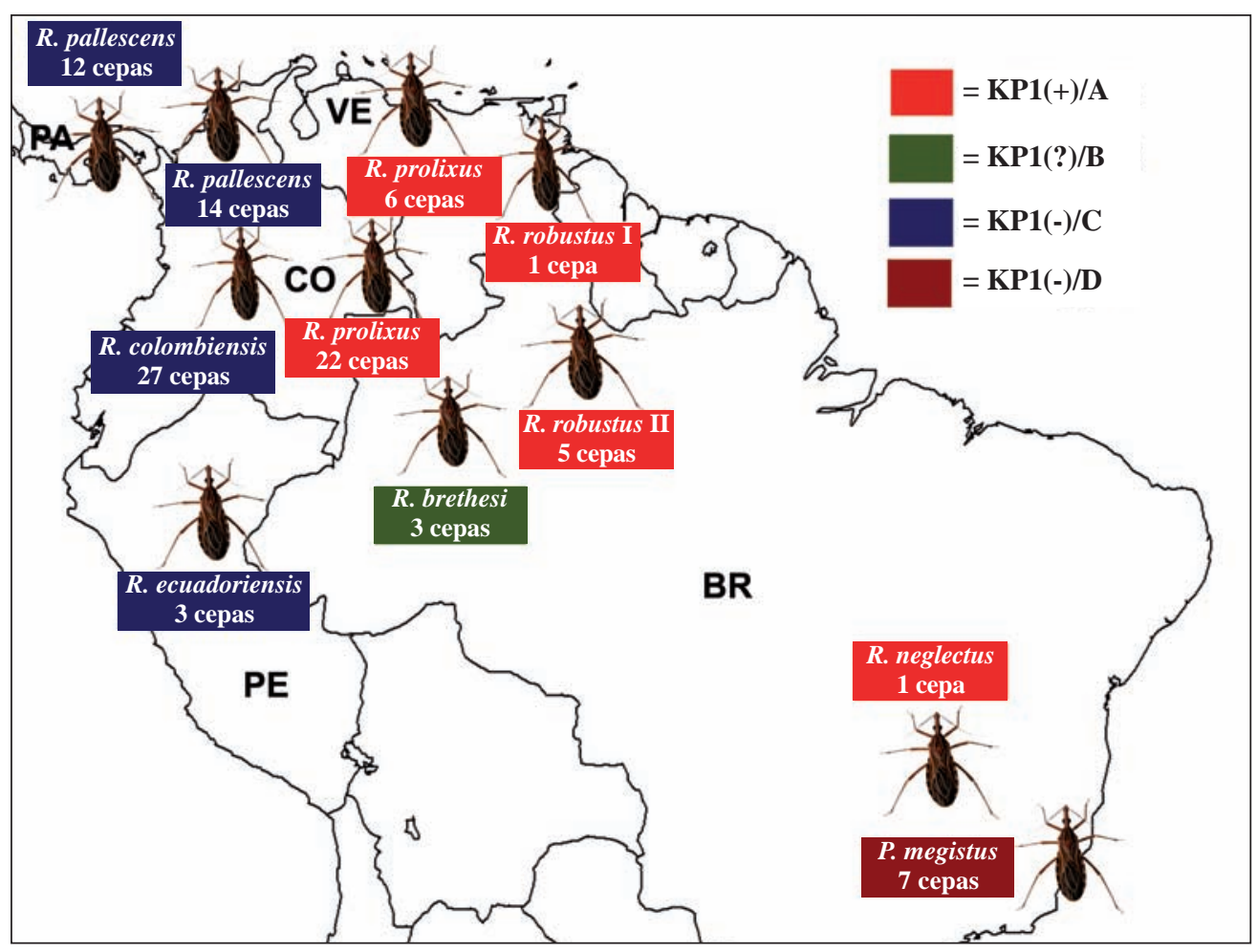

Figura 3. Distribución geográfica de los genotipos de 101 cepas de T. rangeli aisladas de glándulas salivares de diferentes especies de Rhodnius. El genotipo C, aislado de las especies de la cordillera de los Andes $R$. pallescens, $R$. colombiensis y $R$. ecuadoriensis, corresponde al genotipo KP1(-) (KP1(-)/C), mientras que las especies aisladas al oriente de la cordillera de los Andes, $R$. prolixus, $R$. robustus y $R$. neglectus, presentan el genotipo A correspondiente a KP1(+) (KP1+/A). Se desconoce el perfil de kADN del genotipo B. Las cepas de T. rangeli aisladas del intestino de Panstrongylus megistus en el suroriente del Brasil correspondieron al genotipo KP1(-) o D (KP1-/D). (Vallejo, et al., 2002, 2003, 2009a, 2009b; Urrea, et al., 2005, 2011).

nuestro laboratorio que los epimastigotes cortos de las cepas de $T$. rangeli KP1(-) son sensibles a la lisis de la hemolinfa de $R$. prolixus, en contraste con los epimastigotes largos, los cuales son resistentes. Las hemolinfas de $R$. pallescens, $R$. colombiensis, $R$. ecuadoriensis, Triatoma dimidiata y Triatoma maculata no presentaron actividad tripanolítica contra los genotipos KP1(+) o KP1(-).

La presencia de factores tripanolíticos no es el único factor que limita el desarrollo de $T$. rangeli, pues el control para generar una infección productiva de tripomastigotes metacíclicos probablemente se encuentra asociado a la presencia o ausencia de lectinas en la superficie del parásito o en las glándulas salivares que impiden la adhesión, el ingreso y la producción de las formas infectivas (Basseri, et al., 2002). En estudios recientes se ha demostrado que la infección exitosa de T. rangeli para generar tripomastigotes metacíclicos está asociada con la producción de ectofosfatasas en el parásito (G omes, et al., 2008; Dos-Santos, et al., 2012; Dos-Santos, et al., 2013; Freitas-Mesquita \& MeyerFernándes, 2014).

La figura 4 muestra el curso de la infección de $T$. rangeli $\mathrm{KP} 1(+)$ y T. rangeli KP1(-) en diferentes especies de triatominos. Se ha observado que cuando T. rangeli KP1(+) infecta a $R$. prolixus, $R$. robustus o $R$. neglectus, los epimastigotes cortos atraviesan la pared intestinal e ingresan al hemocele, invaden los hemocitos y se multiplican dentro de ellos, y que los epimastigotes cortos luego son reemplazados por epimastigotes largos, los cuales se adhieren a las glándulas salivares y penetran en ellas para finalmente producir los tripomastigotes metacíclicos, que son las formas infectivas para el mamífero (Figura 4B). Otras observaciones inéditas en nuestro laboratorio mostraron que cuando $T$. rangeli $\mathrm{KP} 1(+)$ infecta a $R$. pallescens y $R$. colombiensis, los epimastigotes cortos atraviesan la pared intestinal e ingresan al hemocele, invaden los hemocitos y se multiplican dentro de ellos, y luego se producen epimastigotes largos, que, sin embargo, son incapaces de adherirse a la superficie de las glándulas salivares $\mathrm{y}$, por ende, no se producen tripomastigotes metacíclicos, probablemente por ausencia de lectinas específicas para facilitar la adhesión o por la ausencia de sustratos para las ectofosfatasas del parásito (Figura 4C). En contraste, cuando T. rangeli KP1(-) infecta a $R$. pallescens, $R$. colombiensis y $R$. ecuadoriensis, los epimastigotes cortos atraviesan la pared intestinal e ingresan al hemocele, invaden los hemocitos y se multiplican dentro de ellos, y luego los epimastigotes cortos son reemplazados por epimastigotes largos, los cuales se adhieren a las glándulas 


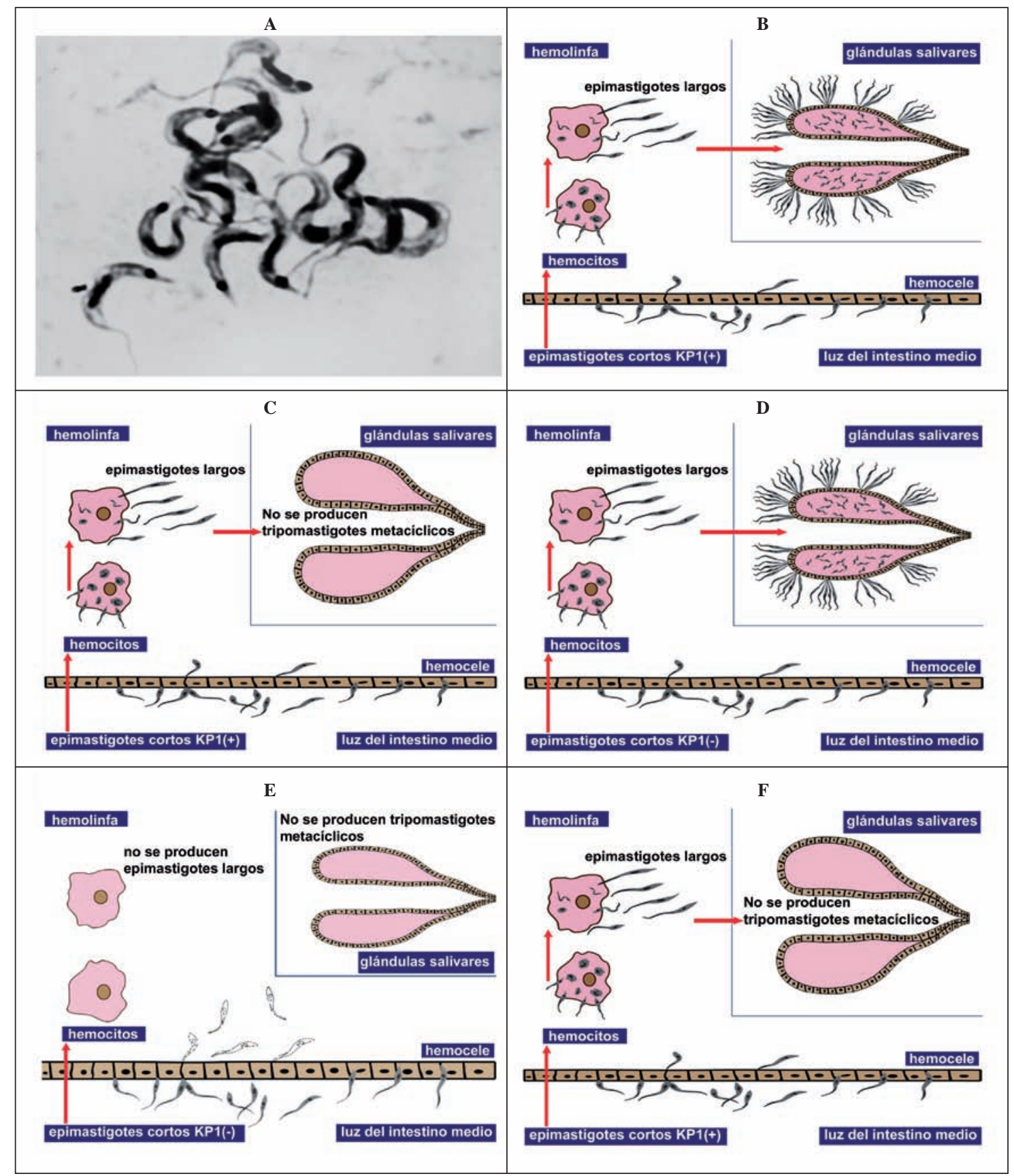

Figura 4. Desarrollo de las infecciones de T. rangeli KP1(+) y T. rangeli KP1(-) en diferentes especies de triatominos. 4A. Tripomastigotes metacíclicos en glándulas salivares de $R$. prolixus, coloreados con Giemsa (x3.000) (Vallejo, 1984). 4B. Infección de T. rangeli KP1(+) con producción de tripomastigotes metacíclicos en $R$. prolixus, $R$. robustus y $R$. neglectus. 4C. Infección de $T$. rangeli KP1(+) en $R$. pallescens y $R$. colombiensis sin producción de tripomastigotes metacíclicos. 4D. Infección de $T$. rangeli KP1(-) con producción de tripomastigotes metacíclicos en $R$. pallescens, $R$. colombiensis y $R$. ecuadoriensis. 4E. Infección de T. rangeli KP1(-) en $R$. prolixus y $R$. robustus sin producción de tripomastigotes metacíclicos debido a que los epimastigotes cortos son lisados inmediatamente después de penetrar en la hemolinfa. 4F. Infección de T. rangeli KP1(+) sin producción de tripomastigotes metacíclicos en Triatoma infestans, Triatoma braziliensis, Triatoma sordida, Triatoma vitticeps y P. megistus (De Stefani- M árquez, et al., 2006).

salivares, penetran en ellas para finalmente producir los tripomastigotes metacíclicos (Figura 4D). Cuando T. rangeli KP1(-) infecta a $R$. prolixus y $R$. robustus, los epimastigotes cortos atraviesan la pared intestinal e ingresan al hemocele e inmediatamente son lisados por factores tripanolíticos presentes en la hemolinfa, de manera que no hay invasión de los hemocitos ni multiplicación dentro de ellos, y no se producen epimastigotes largos ni tripomastigotes metacíclicos 
(Figura 4E). De Stefani-M árquez, et al., (2006) infectaron Triatoma infestans, Triatoma braziliensis, Triatoma sordida, Triatoma vitticeps y Panstrongylus megistus con T. rangeli $\mathrm{KP} 1(+)$ y observaron que los epimastigotes cortos atraviesan la pared intestinal, ingresan al hemocele, invaden los hemocitos y se multiplican dentro de ellos, y luego se producen epimastigotes largos, que, sin embargo, son incapaces de adherirse a la superficie de las glándulas salivares y no producen tripomastigotes metacíclicos (Figura 4F).

El conocimiento sobre los mecanismos bioquímicos o moleculares de la interacción de los tripanosomas y los vectores es aún incompleto, por lo tanto, es necesario identificar los genotipos de $T$. rangeli que son transmitidos por el resto de las 19 especies de Rhodnius, reconocer la estructura química del factor o factores tripanolíticos y comparar la expresión de los factores inmunes en las especies que presentan actividad tripanolítica o impiden la formación de tripomastigotes metacíclicos en las glándulas salivares. También deberá estudiarse el proteoma de las hemolinfas con actividad lítica y sin esta, así como el proteoma de las cepas sensibles o resistentes a la actividad lítica, para confirmar si la transmisión selectiva se extiende a todas las 19 especies de Rhodnius. Nuestro grupo de investigación está adelantando la identificación de proteínas en la hemolinfa de los vectores que podrían estar involucradas en la lisis de los genotipos de T. rangeli (Suárez, et al., 2013). Igualmente, será necesario estudiar si existe alguna relación entre los factores tripanolíticos y la microbiota presente en el intestino del vector.

\section{T. cruzi: interacción parásito-vector}

Debido a que en los últimos años se han acumulado evidencias acerca de la transmisión selectiva de los genotipos de $T$. rangeli en los vectores del género Rhodnius, ha surgido interés en la búsqueda de mecanismos similares en los vectores de $T$. cruzi, los cuales incluyen 148 especies de diferentes géneros de triatominos (J usti, et al., 2014). A diferencia de $T$. rangeli, $T$. cruzi se desarrolla solamente en el intestino del vector $y$, por lo tanto, se enfrenta a factores propios del intestino como enzimas, hemolisinas y factores derivados de la microbiota. Aunque T. cruzi no circula por la hemolinfa del vector, estudios previos publicados por M ello, et al. (1996) mostraron factores tripanolíticos contra $T$. cruzi en la hemolinfa de $R$. prolixus. Por esta razón, nuestro grupo decidió explorar si en la hemolinfa de $R$. prolixus era posible detectar factores tripanolíticos contra las diferentes genotipos de $T$. cruzi (I-VI) previamente descritos por Zingales, et al. (2009, 2012). Utilizando la misma metodología usada para $T$. rangeli, se observó que la hemolinfa de $R$. prolixus presentó actividad lítica contra T. cruzi II y T. cruzi V (Zabala, et al., 2011; Suárez, et al., 2011). Esta actividad tripanolítica también se ha observado en $R$. robustus y se sospecha que está presente en las restantes especies del grupo Robustus: $R$. neglectus, $R$. domesticus y $R$. nasutus.Un factor tripanolítico con actividad para los mismos genotipos de $T$. cruzi se detectó en el estómago de $R$. prolixus (Suárez, et al., 2012). Llama la atención el hecho de que en las áreas geográficas en donde está presente $R$. prolixus predomina el genotipo $T$. cruzi I, el cual es resistente al factor tripanolítico, mientras que $T$. cruzi II, que es sensible, presenta muy baja prevalencia en estas áreas.

Además, en los últimos años se ha logrado identificar el papel de la microbiota del intestino de los vectores como reguladora de la respuesta inmune y de la infección por $T$. cruzi y por $T$. rangeli (C astro, et al., 2012; Vallejo, et al., 2009a). Se ha demostrado que la microbiota de $R$. prolixus produce lisis de $T$. cruzi II pero no de T. cruzi I. Se desconoce si existe alguna relación entre el factor tripanolítico detectado en la hemolinfa de $R$. prolixus y la microbiota intestinal, por lo tanto, es necesario realizar nuevos estudios en esta dirección. En concordancia con lo anterior, en Colombia se ha visto que en las infecciones naturales de Rhodnius predomina $T$. cruzi I sobre T. cruzi II (Vallejo, et al., 2009b). Incluso en las infecciones generales en vectores y vertebrados existe predominio de $T$. cruzi I sobre $T$. cruzi II.

Durante el ciclo de vida de $T$. cruzi el parásito no llega a la hemolinfa del vector, por lo tanto, el factor tripanolítico de la hemolinfa de $R$. prolixus no tendría ningún efecto sobre el parásito. No obstante, se cree que este factor tripanolítico detectado en la hemolinfa podría ser un remanente de la inmunidad innata generada en otros tejidos, como el intestino o el cuerpo graso del vector, contra genotipos de T. cruzi. Estos resultados apoyan la hipótesis sobre la distribución geográfica de los genotipos de T. cruzi y T. rangeli, la cual dependería de la distribución geográfica de los vectores en América Latina.

\section{A nálisis del transcriptoma y del genoma de T. rangeli}

La variabilidad genética de $T$. rangeli, su patogenia para el vector, y el hecho de ser infectivo pero no patógeno para el humano, ha estimulado los estudios de transcripción genómica de este parásito con el objetivo de compararlo con T. cruzi, T. brucei y Leishmania spp y así entender las bases moleculares de la patogenia de estas últimas especies y la ausencia de patogenia de $T$. rangeli para el vertebrado. Grisard, et al., (2010) determinaron el perfil de expresión de $T$. rangeli a partir de la generación de etiquetas de secuencias expresadas (expressed sequence tag, EST) y una técnica denominada ORESTES, que combina los marcos abiertos de lectura (open reading frame, ORF) y las EST. Se analizaron 4.208 secuencias a partir de epimastigotes y tripomastigotes de las cepa SC-58, aislada de Santa Catarina (Brasil) y la Choachí, aislada de Cundinamarca (Colombia), representantes de los genotipos KP1(-) y KP1(+), respectivamente. El análisis comparativo de T. cruzi, T. brucei y Leishmania major permitió asignar las funciones biológicas putativas a la mayoría de las secuencias generadas y la organización de una base de genes anotados de T. rangeli. Aunque no es patógeno para los mamíferos, 
varios genes asociados con virulencia en otras especies de cinetoplástidos patógenos se encontraron en $T$. rangeli, de manera que el significado biológico de la transcripción de estos genes requiere nuevos estudios. Grisard, et al., (2010) estimaron en 8.500 el número de genes de T. rangeli, confirmaron la cercanía filogenética entre $T$. cruzi y $T$. rangeli e identificaron varios elementos transponibles, genes asociados a la expresión mitocondrial y genes específicos de la edición de ARN.

La genómica comparativa es una poderosa herramienta que al ser utilizada entre organismos patógenos filogenéticamente relacionados permite entender los fundamentos bioquímicos relacionados con sus ciclos de vida y las bases moleculares de las enfermedades que causan en el humano. En la actualidad se han secuenciado 25 especies del orden Kinetoplastida, incluidas especies de diferentes géneros de parásitos que infectan insectos y mamíferos, de los cuales los genomas de T. brucei, T. cruzi y L. major se han considerado como modelos. A estos se han añadido nuevas secuencias de genomas de Leishmania y Trypanosoma para estudiar comparativamente la biología compleja de las especies que infectan al humano. La secuenciación reciente del genoma de la cepa SC-58 de T. rangeli KP1(-), o genotipo D, es la primera lograda en esta especie infectiva, no patógena para el humano pero sí para el insecto vector (Stoco, et al., 2014). El genoma haploide de T. rangeli es hasta hoy el más pequeño y menos variable de los tres genomas de tripanosomas que infectan al hombre: T. cruzi, 89,9 Mb, T. brucei, $26 \mathrm{Mb}$, y T. rangeli, $24 \mathrm{Mb}$. El genoma de T. rangeli fue comparado con el de $T$. cruzi, agente causal de la enfermedad de Chagas, y se encontraron diferencias entre las cuales se destacan las siguientes: 1) T. rangeli posee en su genoma menor número de copias de las familias multigénicas consideradas como factores de virulencia en T. cruzi, tales como las proteínas de superficie asociadas a mucinas (mucin-associated surface proteins, MASP), trans-sialidasas y mucinas. Las trans-sialidasas en $T$. cruzi remueven el ácido siálico de la superficie de las células del huésped y lo colocan sobre las moléculas de mucina de la superficie del parásito (F rasch, 2000). Esta transferencia de ácido siálico confiere protección al parásito frente a la activación de la cascada del complemento del huésped. Por el contrario, la sialidasa de $T$. rangeli no permanece unida a la superficie celular del parásito, sino que es liberada abundantemente en el medio de cultivo o en el intestino del vector, razón por la cual se sospecha que esta enzima podría alterar la microbiota intestinal y estar involucrada en la patogenia comprobada de T. rangeli para las especies de Rhodnius (Pereira \& M oss, 1985; Vallejo \& Marinkelle, 1992; Stoco, et al., 2014); 2) $T$. rangeli posee en su genoma un reducido repertorio de genes que codifican enzimas de defensa antioxidante, de manera que $T$. rangeli es altamente susceptible al estrés oxidativo debido a la presencia de un pequeño número de estas enzimas y proteínas de choque térmico; 3) T. rangeli posee ortólogos vestigiales de la maquinaria de ARNi, la cual es insuficiente para constituir una vía bioquímica funcional. Además, el análisis filogenético de genes mitocondriales y nucleares indicó que $T$. rangeli y $T$. cruzi son equidistantes de T. brucei (Stoco, et al., 2014). En general, el análisis comparativo del genoma de $T$. rangeli permitirá un mayor entendimiento de la identidad, evolución, regulación y funcionamiento de los determinantes de la virulencia de los tripanosomas patógenos para los hospederos vertebrados y para los vectores; permitirá, igualmente, desarrollar mejores métodos de diagnóstico, así como la identificación de blancos terapéuticos para el tratamiento y el control de los tripanosomátidos patógenos.

\section{Conclusiones}

Después de la descripción de T. rangeli por Enrique Tejera en Venezuela en 1920, eminentes parasitólogos de Colombia y de América Latina han estudiado durante los últimos 94 años a este fascinante parásito infectivo y no patógeno para los humanos, pero patógeno para los insectos vectores. Los estudios bioquímicos y moleculares realizados en los últimos 25 años han revelado una extensa variabilidad genética del parásito y han mostrado la existencia de diversos genotipos que han evolucionado concertadamente con las especies de Rhodnius, de manera que los vectores han desarrollado mecanismos que impiden la transmisión de algunos genotipos pero permiten la transmisión de otros. Es así como T. rangeli KP1(-) circula en la línea evolutiva Pallescens (R. pallescens, $R$. colombiensis, $R$. ecuadoriensis) y $T$. rangeli $\mathrm{KP} 1(+)$ circula en la línea Robustus ( $R$. prolixus, $R$. robustus, $R$. neglectus). Nuestro grupo de investigación describió la existencia de factores tripanolíticos presentes en la hemolinfa de $R$. prolixus y $R$. robustus que eliminan las cepas de $T$. rangeli KP1(-), pero permiten la transmisión de $T$. rangeli $\mathrm{KP} 1(+)$. Los factores tripanolíticos en la hemolinfa de $R$. prolixus y $R$. robustus también afectan la transmisión de algunos genotipos de $T$. cruzi, lo que sugiere que la dinámica de transmisión de los genotipos de T. cruzi y de $T$. rangeli depende fundamentalmente de las especies de vectores existentes en las diferentes áreas geográficas endémicas para estas dos especies de tripanosomas en América Latina.

Durante los últimos 10 años se han abordado los estudios genómicos y de transcripción de $T$. rangeli con la finalidad de comparar esta información con estudios similares en parásitos patógenos como T. cruzi, T. brucei y Leishmania trópica. Se encontró que el genoma de $T$. rangeli es el más pequeño y el menos repetitivo de los genomas de las tres especies de tripanosomas que infectan al hombre, lo que indica que las familias génicas que responden por la patogenia de T. cruzi para el vertebrado, se expresan en muy baja proporción en el genoma de $T$. rangeli, de forma que este carece del suficiente arsenal de proteínas y enzimas como las MASP y las trans-sialidasas, necesarias para invadir las células del mamífero. A diferencia de T. cruzi, T. rangeli secreta copiosamente las sialidasas en el intestino de 
los vectores, lo cual podría afectar la microbiota bacteriana del insecto y generar un efecto patógeno para el vector (Stoco, et al., 2014). Algunos investigadores consideran que en las regiones donde $T$. rangeli coexiste con $T$. cruzi en los mismos vectores y vertebrados, las infecciones de T. rangeli podrían alterar el curso de la enfermedad de Chagas en los vertebrados (Paláu, et al., 2003), pues se ha observado que la vacunación de ratones, cobayos y perros con $T$. rangeli induce protección inmunitaria contra cepas virulentas de $T$. cruzi (Basso, et al., 2007; Basso, et al., 2008, 2014), lo que abre nuevas perspectivas para el control de la enfermedad de Chagas en los reservorios domésticos y, probablemente, en los humanos.

\section{Agradecimientos}

Este trabajo está dedicado a la memoria del Dr. Cornelis Johannes Marinkelle (q.e.p.d.), bajo cuya dirección Gustavo Adolfo Vallejo, autor principal del presente artículo, inició en 1981 los estudios de $T$. rangeli en el CIMPAT de la Universidad de los Andes de Bogotá, y con quien mantuvo durante los siguientes 31 años estimulantes discusiones que motivaron y orientaron la búsqueda de respuestas al enigmático comportamiento de este parásito infectivo y no patógeno para el humano.

Información suplementaria de agradecimientos, http://www. raccefyn.co/index.php/raccefyn/article/downloadSuppFile/ $143 / 623$

\section{Conflicto de intereses}

Los autores declaran que no existen conflictos de intereses relacionados con el contenido del presente artículo.

\section{Bibliografía}

Abad-Franch, F., M onteiro, F.A., J aramillo, O. N., G urgelG onçalves, R ., Dias, F.B., Diotaiuti, L. 2009. Ecology, evolution, and the long-term surveillance of vector-borne Chagas disease: A multi-scale appraisal of the tribe Rhodniini (Triatominae). Acta Tropica. 110 (2-3): 159-177. doi: 10.1016/j.actatropica.2008.06.005.

A bad-Franch, F., Pavan, M.G., J aramillo-O, N., Palomeque, F.S., Dale, C., Chaverra, D., Monteiro, F.A. 2013. Rhodnius barretti, a new species of Triatominae (Hemiptera: Reduviidae) from western Amazonia. Mem Inst Oswaldo Cruz. 108 Suppl 1: 92-99. doi: 10.1590/0074-0276130434.

Azambuja, P. \& G arcía, E.S. 2005. Trypanosoma rangeli interactions within the vector Rhodnius prolixus: A mini review. Memorias do Instituto Oswaldo Cruz. 100 (5): 567-572.

Azambuja, P., R atcliffe, N.A., García, E.S. 2005. Towards an understanding of the interactions of Trypanosoma cruzi and Trypanosoma rangeli within the reduviid insect host Rhodnius prolixus. Acad Bras Cienc. 77 (3): 397-404.

Basseri, H.R ., Tew, I.F., R atcliffe, N.A. 2002. Identification and distribution of carbohydrate moieties on the salivary glands of Rhodnius prolixus and their possible involvement in attachment/invasion by Trypanosoma rangeli. Experimental Parasitology. 100: 226-234.

Basso, B., C astro, I., Introini, V., Gil, P., Truyens, C., M oretti, E. 2007. Vaccination with Trypanosoma rangeli reduces the infectiousness of dogs experimentally infected with Trypanosoma cruzi. Vaccine. 25 (19): 3855-3858.

Basso, B., Moretti, E., Fretes, R. 2008. Vaccination with epimastigotes of different strains of Trypanosoma rangeli protects mice against Trypanosoma cruzi infection. Mem Inst Oswaldo Cruz. 103 (4): 370-374.

Basso, B., Moretti, E., Fretes, R. 2014. Vaccination with Trypanosoma rangeli induces resistance of guinea pigs to virulent Trypanosoma cruzi. Vet Immunol Immunopathol. 157 (1-2): 119-123. doi: 10.1016/j.vetimm.2013.10.011.

Castro, D.P., M oraes, C.S., G onzález, M.S., R atcliffe, N.A., Azambuja, P., García, E.S. 2012. Trypanosoma cruzi Immune Response Modulation Decreases Microbiota in Rhodnius prolixus Gut and Is Crucial for Parasite Survival and Development. PLoS ONE. 7 (5): e36591.

D'Alessandro, A. 1976. Biology of Trypanosoma (Herpetosoma) rangeli Tejera, 1920. In WHR Lumsden \& DA Evans (editors), Biology of Kinetoplastida, Vol. 1, Academic Press, London, New York and San Francisco, pp. 327-493.

D'Alessandro, A., Hincapié, 0. 1986. Rhodnius neivai: A new experimental vector of Trypanosoma rangeli. Am J Trop Med Hyg. 35: 512-514.

D'A lessandro-Bacigalupo, A., G ore-Saravia, N. 1999. Trypanosoma rangeli. In: Gilles Herbert, M. (Ed.), Protozoal Diseases. Oxford University Press, Oxford, pp. 398-412.

da Rosa, J .A., M endonça, V.J ., G ardim, S., de Carvalho, D.B., de Oliveira, J ., Nascimento, J .D., Pinotti, H., Pinto, M .C., Cilense, M ., Galvão, C ., Barata, J .M. 2014. Study of the external female genitalia of 14 Rhodnius species (Hemiptera, Reduviidae, Triatominae) using scanning electron microscopy. Parasit Vectors. 7: 17. doi: 10.1186/1756-3305-7-17.

De Stefani-M árquez, M.D., R odrigues-0ttaiano, C ., M ônica O liveira, R ., Pedrosa, A.L ., C abrine-Santos, M., LagesSilva, E ., R amírez, L .E . 2006. Susceptibility of different triatomine species to Trypanosoma rangeli experimental infection. Vector Borne Zoonotic Dis. 6 (1): 50-56.

Dos-Santos, A .L .A ., D ick, C .F., Alves-Bezerra, M ., Silveira, T.S., Paes, L .S., G ondim, K.C ., M eyer-Fernandes, J .R. 2012. Interaction between Trypanosoma rangeli and the Rhodnius prolixus salivary gland depends on the phosphotyrosine ecto-phosphatase activity of the parasite. International Journal for Parasitology. 42: 819-827.

Dos-Santos, A.L.A., Dick, C.F., Silveira, T.S., Fonseca-deSouza, A.L., M eyer-Fernandes, J .R. 2013. Trypanosoma rangeli: An alkaline ecto-phosphatase activity is involved with survival and growth of the parasite. Experimental Parasitology. 135: 459-465.

Frasch, A.C.C. 2000. Functional diversity in the trans-sialidase and mucin families in Trypanosoma cruzi. Parasitol Today. 16: 282-286. 
Freitas-M esquita, A.L ., \& M eyer-Fernandes, J .R . 2014. Ectonucleotidases and ecto-phosphatases from Leishmania and Trypanosoma Parasites. Proteins and proteomics of Leishmania and Trypanosoma Subcellular Biochemistry. 74: $217-252$.

Garcia, E.S., Castro, D.P., Figueiredo, M.B., Genta, F.A., Azambuja, P. 2009. Trypanosoma rangeli: A new perspective for studying the modulation of immune reactions of Rhodnius prolixus. Parasit Vectors. 2 (1): 33. doi: 10.1186/1756-3305-2-33

Garcia, E .S., G enta, F.A., de Azambuja, P., Schaub, G .A. 2010. Interactions between intestinal compounds of triatomines and Trypanosoma cruzi. Trends Parasitol. 26 (10): 499-505. doi: 10.1016/j.pt.2010.07.003

G arcia, E.S., C astro, D.P., Figueiredo, M .B., Azambuja, P. 2012. Parasite-mediated interactions within the insect vector: Trypanosoma rangeli strategies. Parasit Vectors. 5: 105. doi: 10.1186/1756-3305-5-105

Gomes, S.A.O., Fonseca de Souza, A.L., K iffer-M oreira, T., Dick, C.F., dos Santos, A.L.A., M eyer-Fernandes, J.R. 2008. Ecto-phosphatase activity on the external surface of Rhodnius prolixus salivary glands: Modulation by carbohydrates and Trypanosoma rangeli. Acta Tropica. 106: $137-142$.

Grisard, E.C., Stoco, P.H., Wagner, G., Sincero, T.C.M., Rotava, G., R odrigues, J.B., Snoeijer, C.Q., K oerich, L .B., Sperandio, M.M., Bayer-Santos, E ., Fragoso, S.P., G oldenberg, S., Triana, O., Vallejo, G.A., Tyler, K.M., Dávila, A .M .R ., Steindel, M . 2010. Transcriptomic analyses of the avirulent protozoan parasite Trypanosoma rangeli. Molecular and Biochemical Parasitology. 174: 18-25.

Groot, H., Renjifo, S., Uribe, C. 1950. Nota preliminar sobre inoculación a un voluntario humano con Trypanosoma sp. (Ariarii). An. Soc. Biol. Bogotá. 4: 99-103.

G root, H., R enjifo, S., Uribe, C . 1951. Trypanosoma ariarii. N. sp. from man, found in Colombia. Am. J. Trop. Med. 31: 673-691.

Groot, H. 1952. Further observations on Trypanosoma ariarii in Colombia. Am. J. Trop. Med. Hyg. 1 (4): 585-592.

Groot, H. 1954. Estudios sobre los tripanosomas humanos clasificados como $T$. rangeli con especial referencia a su evolución en Rhodnius prolixus y a su comparación con $T$. ariarii. An. Soc. Biol. Bogotá. 6 (3): 109-126.

Guhl, F. \& M arinkelle, C.J . 1982. Antibodies against Trypanosoma cruzi in mice infected with T. rangeli. Ann. Trop. Med. Parasitol. 76: 361.

G uhl, F., H udson, L., M arinkelle, C.J ., M organ, S., J aramillo, C. 1985. Antibody response to experimental Trypanosoma rangeli infection and its implications for immunodiagnosis of South American trypanosomiasis. Acta Trop 42: 311-318.

Guhl, F. \& Vallejo, G.A. 2003. Trypanosoma (Herpetosoma) rangeli Tejera, 1920: An updated review. Memorias do Instituto Oswaldo Cruz. 98 (4): 435-442.

J usti, S.A., R usso, C .A., Mallet, J .R ., O bara, M.T., G alvão, C. 2014. Molecular phylogeny of Triatomini (Hemiptera: Reduviidae: Triatominae). Parasit. Vectors. 7: 149. doi: 10.1186/1756-3305-7-149
M achado, P.E., Eger-M angrich, I., R osa, G., K oerich, L.B., G risard, E.C., Steindel, M. 2001. Differential susceptibility of triatominae of the genus Rhodnius to Trypanosoma rangeli strains from different geographical origins. Int $\mathrm{J}$ Parasitol. 31: 631- 633.

Maia da Silva, F., Rodrigues, A.C., Campaner, M., Takata, C.S.A., Brigido, M.C., J unqueira, A.C.V., C oura, J .R., Takeda, G.F., Shaw, J.J., Texeira, M.M.G. (2004a). Randomly amplified polymorphic DNA analysis of Trypanosoma rangeli and allied species from human, monkeys and other sylvatic mammals of the Brazilian Amazon disclosed a new group and a species-specific marker. Parasitology. 128: $283-294$.

M aia da Silva, F., Noyes, H., C ampaner, M., J unqueira, A.C.V., C oura, J .R ., A ñez, N., Shaw, J J ., Stevens, J .R ., Teixeira, M.M.G . 2004b. Phylogeny, taxonomy and grouping of Trypanosoma rangeli isolates from man, triatomines and sylvatic mammals from widespread geographical origin based on SSU and ITS ribosomal sequences. Parasitology. 129: $549-561$.

M aia da Silva, F., J unqueira, A.C ., C ampaner, M ., R odrigues, A.C., Crisante, G., Ramírez, L.E., Caballero, Z.C., M onteiro, F.A., C oura, J .R., Añez, N., Teixeira, M .M. 2007. Comparative phylogeography of Trypanosoma rangeli and Rhodnius (Hemiptera: Reduviidae) supports a long coexistence of parasite lineages and their sympatric vectors. Mol. Ecol. 16: 3361-3373.

Maia da Silva, F., Marcili, A., L ima, L ., C avazzana J r., M., Ortiz, P.A., Campaner, M., Takeda, G.F., Paiva, F., Nunes, V.L .B., Camargo, E.P., Teixeira, M.M.G. 2009. Trypanosoma rangeli isolates of bats from Central Brazil: Genotyping and phylogenetic analysis enable description of a new lineage using spliced-leader gene sequences. Acta Trop. 109: 199-207.

M arinkelle, C J . 1968a. Triatoma dimidiata capitata, a natural vector of Trypanosoma rangeli in Colombia. Rev Biol Trop. 15: 203-205.

Marinkelle, C.J . 1968b. Pathogenicity of Trypanosoma rangeli for Rhodnius prolixus Stal in nature. J Med Entomol 5: 497- 499.

M ello, C.B., Azambuja, P., G arcia, E.S., R atcliffe, N.A. 1996. Differential in vitro and in vivo behavior of three strains of Trypanosoma cruzi in the gut and hemolymph of Rhodnius prolixus. Exp Parasitol. 82 (2): 112-121.

Meneguetti, D.U.O., Soares, E.B., C ampaner, M., C amargo, L.M.A. 2014. First report of Rhodnius montenegrensis (Hemiptera: Reduviidae: Triatominae) infection by Trypanosoma rangeli. Revista da Sociedade Brasileira de Medicina Tropical. 47 (3): 374-376.doi:10.1590/0037-8682-0179-2013

Organización Panamericana de Salud. 2007. Estimación cuantitativa de la enfermedad de Chagas en las Américas. Montevideo: (OPS/HDM/CD/425-06); pp. 6-17.

Organización Mundial de la Salud. 2014. La enfermedad de Chagas (Tripanosomiasis Americana). Nota descriptiva $\mathrm{N}^{\circ}$ 340. 2014. Fecha de consulta: 4 de septiembre de 2014. Disponible en: http://www.who.int/mediacentre/factsheets/ fs340/es/index.html 
Paláu, M.T., M ejía, A.J., Vergara, U. Zúñiga, C.A. 2003. Action of Trypanosoma rangeli in Infections with virulent Trypanosoma cruzi populations. Memorias do Instituto Oswaldo Cruz. 98 (4): 543-548.

Pereira, M.E.A. \& M oss, D. 1985. Neuraminidase activity in Trypanosoma rangeli. Molecular and Biochemical Parasitology. 15: 95-103.

Pulido, X.C., Pérez, G., Vallejo, G .A. 2008. Preliminary characterization of a Rhodnius prolixus hemolymph trypanolytic protein, this being a determinant of Trypanosoma rangeli $\mathrm{KP} 1(+)$ and KP1(-) subpopulations' vectorial ability. Memorias do Instituto Oawaldo Cruz. 103 (2): 172-179.

Salazar-A ntón, F., Urrea, D.A., G uhl, F., A révalo, C., Azofeifa G., Urbina A., Blandón-Naranjo, M. Sousa, O.E., Zeledón, R., Vallejo, G.A. 2009. Trypańosoma rangeli genotypes association with Rhodnius prolixus and $R$. pallescens allopatric distribution in Central America. Infection, Genetics and Evolution. 9: 1306-1310.

Schaub, G.A. 2009. Interactions of Trypanosomatids and Triatomines. In Stephen J. Simpson and Jerome Casas: Advances in Insect Physiology, Vol. 37, Burlington: Academic Press. pp.177-242. ISBN: 978-0-12-374829-4.

Schofield, C.J. \& Dujardin, J.P. 1999. Theories on the evolution of Rhodnius. Actualidades Biológicas. 21: 183-197.

Stoco, P.H., Wagner, G., Talavera-L opez, C ., G erber, A., Z aha, A., Thompson, C.E., Bartholomeu, D.C ., L ückemeyer, D.D., Bahia, D., Loreto, E., Prestes, E.B., Lima, F.M., Rodrigues-Luiz, G., Vallejo, G.A., Filho, J .F., Schenkman, S., M onteiro, K.M., Tyler, K.M., Almeida, L .G ., Ortiz, M .F., C hiurillo, M .A., M oraes, M.H., C unha Ode. L., Mendonça-Neto, R., Silva, R., Teixeira, S.M., M urta, S.M ., Sincero, T.C., M endes, T.A ., U rmenyi, T.P., Silva, V.G ., Da Rocha, W.D., Andersson, B., Romanha, A.J ., Steindel, M ., Vasconcelos, A.T., G risard, E .C . 2014. Genome of the Avirulent Human-Infective TrypanosomeTrypanosoma rangeli. PLoS Negl. Trop. Dis. 8 (9): e3176. doi: 10.1371/journal.pntd.0003176

Suárez, Y., Vega, N., Z abala, D., G ranada, Y., R oa, L ., G aitán X., Alvarado, U., Serrato C., M ontilla, M., Texeira, M., Urrea, D., Villa, L ., C arranza, J .C., Vallejo G .A. 2011. Factores tripanolíticos de la hemolinfa de Rhodnius prolixus que actúan sobre Trypanosoma cruzi II, V y VI, pero no sobre T. cruzi I, sugieren una transmisión selectiva de genotipos en Colombia, Venezuela y Centroamérica. Memorias del XX Congreso Latinoamericano de Parasitología Tropical y XV Congreso de Parasitología y Medicina Tropical. Biomédica. Bogotá Colombia. Octubre de 2011.

Suárez, Y., G aitán, X., R oa, L .A., C arranza, J .C ., Vallejo, G .A. 2012. La presencia de factores tripanolíticos en el estómago de Rhodnius prolixus contribuye a la transmisión selectiva de genotipos de Trypanosoma cruzi y T. rangeli en América Latina. Memorias del XLVII Congreso Nacional de Ciencias Biológicas. Santiago de Cali, Colombia, octubre de 2012.

Suárez Y., G onzález C.I., Díaz, M.L., O laya, J L., G utiérrez, S., Carranza, J.C., Vallejo, G.A. 2013. Análisis proteómico de diferentes especies de triatominos para identificar factores asociados a la transmisión selectiva de genotipos de Trypanosoma cruzi y T. rangeli en América Latina. Memorias del XXI Congreso de la Federación Latinoamericana de Parasitología (FLAP), Guayaquil, Ecuador. Octubre de 2013.

Uribe, C. 1929. Infección del Rhodnius prolixus Stahl por Trypanosoma cruzi y Trypanosoma rangeli. Revista MédicoQuirúrgica de los Hospitales. Bogotá. 3 (20): 133-137.

Urrea, D.A., Carranza, J.C., C uba-Cuba, C.A., GurgelGonçalves, R., Guhl, F., Schofield, C.J., Triana O., Vallejo, G.A. 2005. Molecular characterisation of Trypanosoma rangeli strains isolated from Rhodnius ecuadoriensis in Perú, $R$. colombiensis in Colombia and R. pallescens in Panamá, supports a co-evolutionary association between parasites and vectors. Infection, Genetics and Evolution. 5 (2): 123-129.

Urrea, D.A., Herrera, C.P., Falla, A., Carranza, J.C., CubaCuba, C., Triana-C hávez, O., Grisard, E.C., Guhl, F., Vallejo, G.A. 2011. Sequence analysis of the splicedleader intergenic region (SL-IR) and random amplified polymorphic DNA (RAPD) of Trypanosoma rangeli strains isolated from Rhodnius ecuadoriensis, $R$. colombiensis, $R$. pallescens and $R$. prolixus suggests a degree of co-evolution between parasites and vectors. Acta Tropica. 120: 59-66.

Vallejo, G .A. 1984. Diferenciación entre las formas de desarrollo de Trypanosoma cruzi y las formas de desarrollo de Trypanosoma rangeli en el intestino del vector Rhodnius prolixus. Tesis para optar el título de Magister en Microbiología. Universidad de los Andes de Bogotá. 335 pp.

Vallejo, G.A., Marinkelle, C.J., Guhl, F., de Sánchez, N. 1988. Comportamiento de la infección y diferenciación morfológica entre Trypanosoma cruzi y T. rangeli en el intestino del vector Rhodnius prolixus. Revista Brasileira de Biología. 48 (3): 577-587.

Vallejo, G.A. \& Marinkelle, C.J. 1992. Estudio sobre la presencia de neuraminidasa en Rhodnius prolixus infectado con Trypanosoma rangeli. Revista Latinoamericana de Microbiología. 34: 91-94.

Vallejo, G .A., G uhl, F., C arranza, J .C ., L ozano, L .E ., Sánchez, J .L ., J aramillo, J .C ., G ualtero, D., C astañeda, N., Silva, J.C., Steindel, M. 2002. KDNA markers define two major Trypanosoma rangeli lineages in Latin-America. Acta Tropica. 81 (1): 77-82.

Vallejo, G .A., G uhl, F., Carranza, J .C., M oreno, J ., Triana, 0 ., Grisard, E. 2003. Parity between kinetoplast DNA and miniexon gene sequences supports either clonal evolution or speciation in Trypanosoma rangeli strains isolated from Rhodnius colombiensis, R. pallescens and R. prolixus in Colombia. Infection, Genetics and Evolution. 3 (1): 39-45.

Vallejo, G.A., Guhl, F., Schaub, G.A. 2009a. TriatominaeTrypanosoma cruzi/T. rangeli: Vector-Parasite Interactions. Acta Tropica. 110: 137-147.

Vallejo, G .A., G uhl, F., C arranza, J .C ., Herrera, C ., Urrea, D.A., Falla, A., Zabala, D., Villa, L.M. 2009b. Trypanosoma cruzi population variability in Colombia: Possible coevolution in different vector species. Revista de la Sociedad Brasilera de Medicina Tropical. 42 (Suplemento II): 27-34. 
Zabala, D, Carranza, J.C., Urrea, D.A., G uhl, F., J aramillo, N., Teixeira, M .M., Vallejo, G .A. 2011. Respuesta inmune diferencial de triatominos contra Trypanosoma cruzi y $T$. rangeli. Rev. Asoc. Col. Cienc. Biol. (Col.). 23: 134-143.

Zingales, B., Andrade, S.G., Briones, M.R., Campbell, D.A., Chiari, E., Fernandes, O., Guhl, F., Lages-Silva, E., M acedo, A.M ., M achado, C.R ., M iles, M .A., R omanha, A.J ., Sturm, N.R ., Tibayrenc, M ,, Schijman, A.G . 2009. A new consensus for Trypanosoma cruzi intraspecific nomenclature: Second revision meeting recommends Tcl to TcVI. Mem Inst Oswaldo Cruz 104: 1051-1054.

Zingales, B., M iles, M .A ., C ampbell, D.A., Tibayrenc, M ., M acedo, A.M., Teixeira, M.M., Schijman, A.G., Llewellyn, M.S., L ages-Silva, E ., Machado, C.R ., Andrade, S.G., Sturm, N.R. 2012. The revised Trypanosoma cruzi subspecific nomenclature: Rationale, epidemiological relevance and research applications. Infect. Genet. Evol. 12 (2): 240-53. doi: 10.1016/j.meegid.2011.12.009. 